ARTICLE

DOI: $10.1038 / s 41467-017-00704-3$

\title{
The Ino80 complex mediates epigenetic centromere propagation via active removal of histone $\mathrm{H} 3$
}

\author{
Eun Shik Choi (1) ${ }^{1}$, Youngseo Cheon (1D ${ }^{1}$, Keunsoo Kang $^{2}$ \& Daeyoup Lee
}

The centromere is the chromosomal locus at which the kinetochore is assembled to direct chromosome segregation. The histone H3 variant, centromere protein A (CENP-A), is known to epigenetically mark active centromeres, but the mechanism by which CENP-A propagates at the centromere, replacing histone $\mathrm{H} 3$, remains poorly understood. Using fission yeast, here we show that the Ino80 adenosine triphosphate (ATP)-dependent chromatin-remodeling complex, which removes histone $\mathrm{H} 3$-containing nucleosomes from associated chromatin, promotes CENP-A ${ }^{\mathrm{Cnp} 1}$ chromatin assembly at the centromere in a redundant manner with another chromatin-remodeling factor $\mathrm{Chd1}^{\mathrm{Hrp} 1}$. CENP-A ${ }^{\mathrm{Cnp} 1}$ chromatin actively recruits the Ino80 complex to centromeres to elicit eviction of histone H3-containing nucleosomes. Artificial targeting of Ino80 subunits to a non-centromeric DNA sequence placed in a native centromere enhances the spreading of CENP-A ${ }^{C n p 1}$ chromatin into the non-centromeric DNA. Based on these results, we propose that CENP-A ${ }^{\text {Cnp1 }}$ chromatin employs the Ino80 complex to mediate the replacement of histone $\mathrm{H} 3$ with $C E N P-A^{C n p 1}$, and thereby reinforces itself.

\footnotetext{
${ }^{1}$ Department of Biological Sciences, Korea Advanced Institute of Science and Technology, Daejeon 34141, South Korea. ${ }^{2}$ Department of Microbiology, Dankook University, Cheonan, Chungnam 31116, South Korea. Eun Shik Choi and Youngseo Cheon contributed equally to this work. Correspondence and requests for materials should be addressed to D.L. (email: daeyoup@kaist.ac.kr)
} 
n most eukaryotes, centromeres are epigenetically specified by chromatin containing the centromere-specific histone $\mathrm{H} 3$ variant, centromere protein A (CENP-A). A model of self-propagating centromeres suggests that pre-established CENP-A chromatin at the centromeres is sufficient to direct replenishment of new CENP-A during the cell cycle ${ }^{1-5}$. At human centromeres, CENP-A replenishment occurs during G1 phase $^{6}$ and is preceded by the replication-independent loss of histone H3.3 incorporated during $\mathrm{S}$ phase ${ }^{7}$. This suggests that centromeres possess a mechanism that actively removes histone H3.3 to make room for CENP-A deposition. The fission yeast, Schizosaccharomyces pombe, has epigenetically defined "regional centromeres" whose chromatin and protein compositions are similar to those of their human counterparts ${ }^{8-10}$. In fission yeast, CENP-A ${ }^{\text {Cnp1 }}$ replenishment at centromeres occurs during G2 phase in a DNA replication-independent manner ${ }^{11}$, and histone $\mathrm{H} 3$ competes with CENP-A ${ }^{\mathrm{Cnpl}}$ for incorporation into centromeres ${ }^{12}$. This suggests that active removal of histone $\mathrm{H} 3$ may be a conserved mechanism required for CENP-A deposition at fission yeast centromeres, and makes the fission yeast an attractive genetic model for studying how histone $\mathrm{H} 3$ is replaced by CENP-A at regional centromeres.

Adenosine triphosphate (ATP)-dependent chromatinremodeling factors can disassemble nucleosomes and, in some cases, induce the exchange of histone variants ${ }^{13-17}$. Thus, they are good candidates to be factors through which centromeres induce the removal of histone $\mathrm{H} 3$. In support of this possibility, ATPdependent chromatin-remodeling factors have been implicated in the assembly or maintenance of centromeric chromatin. In Saccharomyces cerevisiae, which has genetically defined "point centromeres" consisting of a single CENP-A Cse4 nucleosome per centromere, the Ino80 (inositol-requiring mutant 80) ATPdependent chromatin-remodeling complex acts to maintain normal chromatin structure at centromeres ${ }^{18}$. However, the Ino80 complex in $S$. cerevisiae contributes to the integrity of pericentric chromatin rather than the assembly of CENP-A ${ }^{\mathrm{Cse} 4}$ a
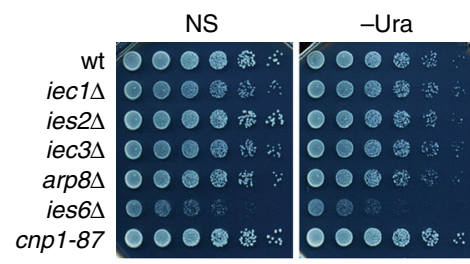

Longer incubation

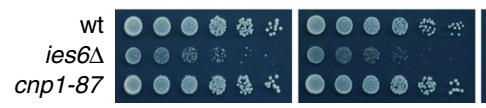

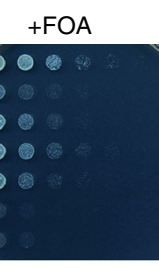

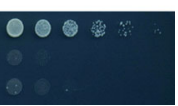

b

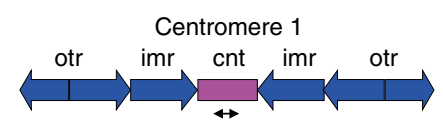

ChIP: $\alpha$ CENP-A ${ }^{\text {Cnp1 }}$

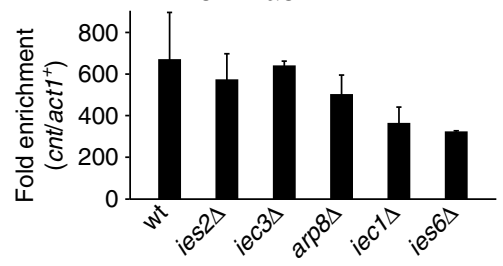

C
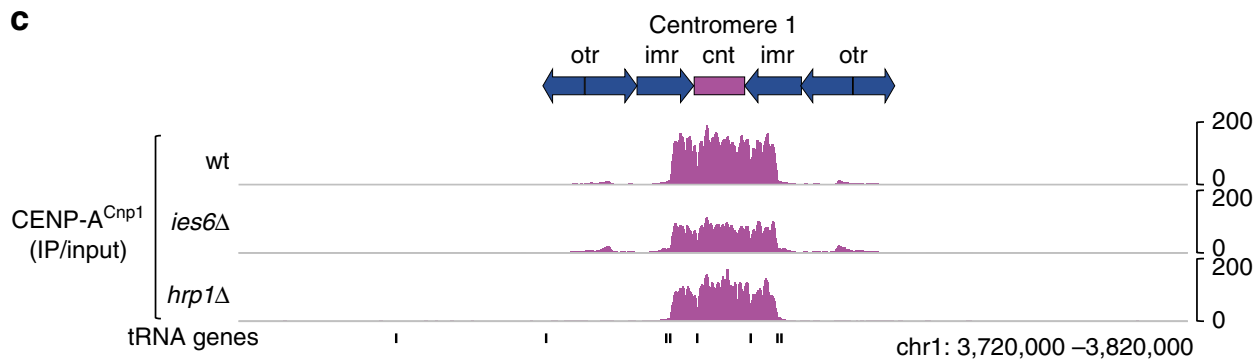

d

e
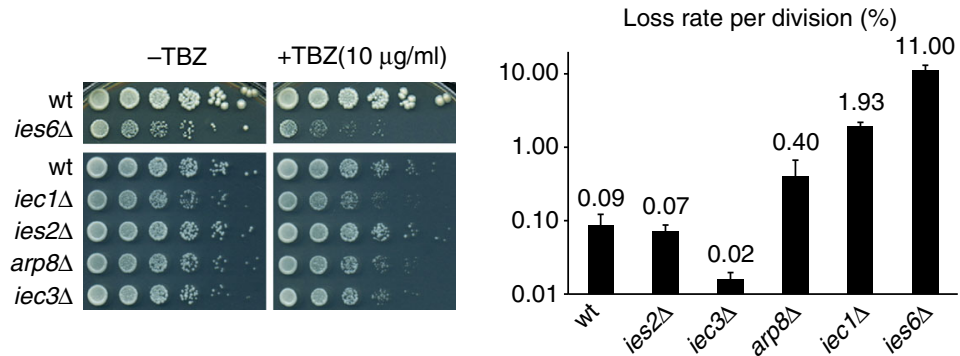

Fig. 1 Defects in the Ino80 complex impair assembly and function of centromeric chromatin. a Silencing of ura $4^{+}$inserted at centromere 1 (cnt1:ura $4^{+}$) of wild-type (wt) and those harboring mutants of the Ino80 complex. The ies6 $\Delta$, wt, and CENP-A ${ }^{\text {Cnp1 }}$ mutant (cnp1-87) cells were incubated for one additional day (denoted by "longer incubation") to ensure full growth. Serial dilutions (fivefold) of cells were spotted onto non-selective (NS), uracil-depleted (-Ura), or FOA-containing ( $+\mathrm{FOA}$ ) medium at $30^{\circ} \mathrm{C}$. FOA was used to kill ura $4^{+}$-expressing cells. $\mathbf{b}$ Schematic of centromere 1 (top), indicating the central core (cnt), innermost repeat (imr), and outer repeats (otr/dg-dh). The primer pairs corresponding to $\mathrm{cnt}$ are indicated below. ChIP analysis for CENP-A ${ }^{\mathrm{Cnp} 1}$ in the indicated cells grown at $30^{\circ} \mathrm{C}$ (bottom). Fold enrichment was calculated by comparing the cnt/act $1^{+}$ratio between the IP and input DNA. Data indicate the mean \pm s.d. (error bars) of three biological replicates. c Genome browser view of centromere 1 showing the ChIP-Seq profiles of CENP-A ${ }^{\text {Cnp1 }}$. Fold

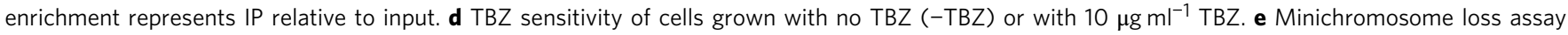
monitoring the loss of non-essential chromosome 16 (Ch16) during cell division. Cells $(618-4,837)$ from three independent colonies for each strain were analyzed to estimate the proportion of cells in the colony containing the minichromosome (see Methods for more detail). Data indicate the mean loss rate \pm s.d. (error bars) 
a

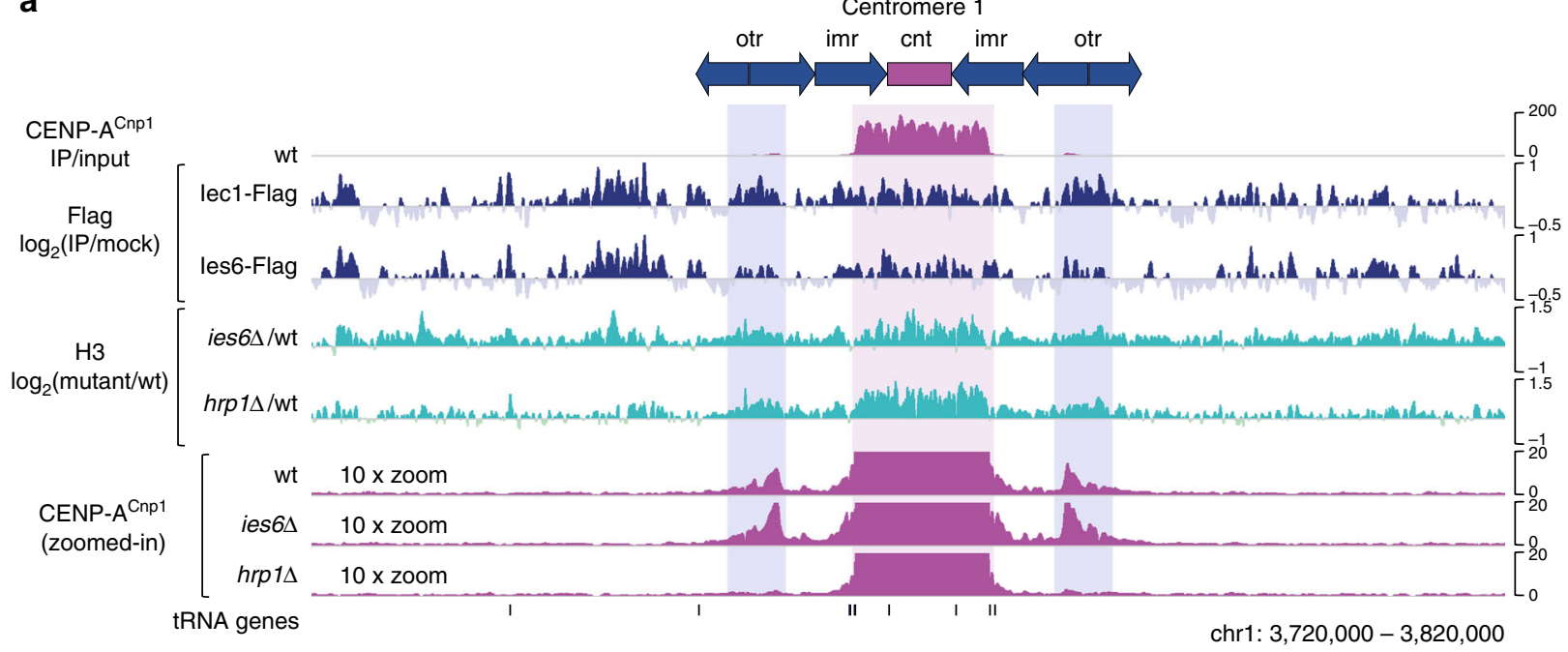

\section{b}

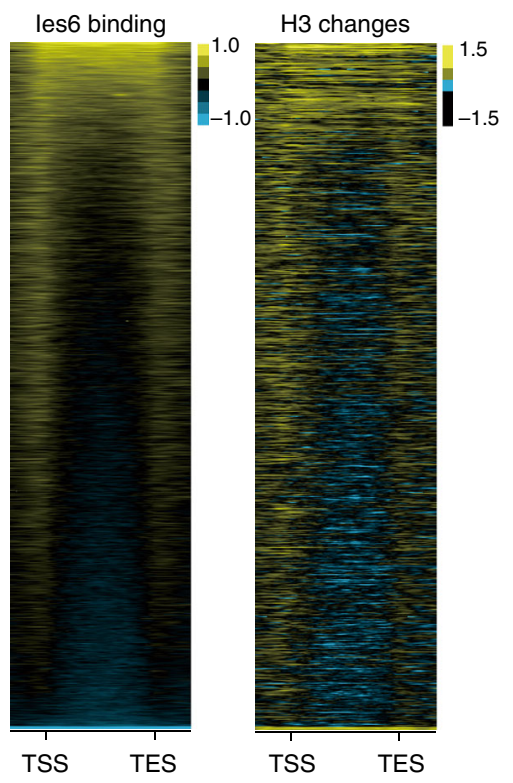

C
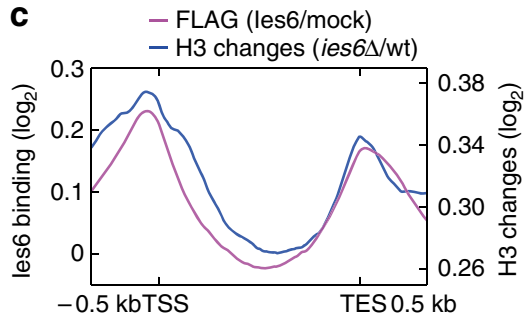

d

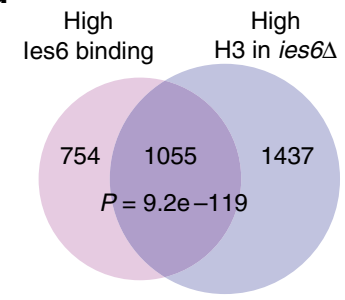

e Non-centromeric euchromatin

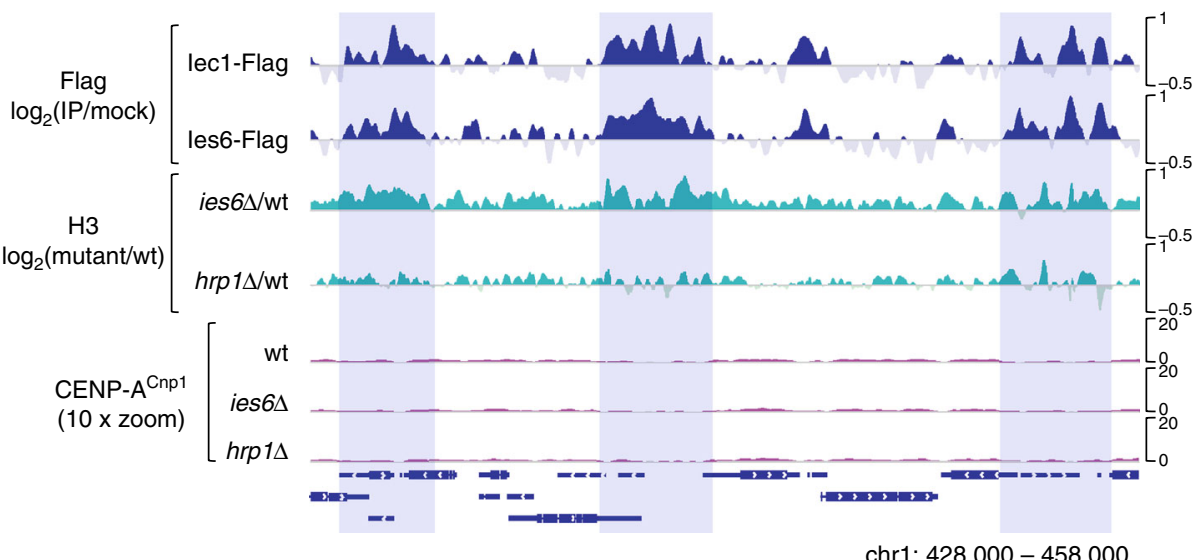

chr1: $428,000-458,000$

Fig. 2 The Ino80 complex induces removal of histone H3 from associated chromatin. a ChIP-Seq profiles of CENP-A Cnp1, lec1-5×Flag, and les6-5×Flag in wt cells, and the histone $\mathrm{H} 3$ changes in ies $6 \Delta$ and hrp1 $1 \Delta$ cells relative to wt at centromere 1. The ChIP enrichments for Flag-tagged Ino80 subunits were normalized to those in mock (no tag) cells. The H3 ChIP-Seq experiments were performed and analyzed using wild-type S. cerevisiae cells as external spikein controls (see Methods for more detail). Magnified $(\times 10)$ views of CENP-A ${ }^{\text {Cnp1 }}$ are shown below. The magenta and blue vertical shadings indicate the major CENP-A ${ }^{C n p 1}$ peaks in the central domain and the minor CENP-A ${ }^{C n p 1}$ peaks in the heterochromatin, respectively. $\mathbf{b}$ Heatmaps representing les6 binding in wt cells (left) and $\mathrm{H} 3$ occupancy changes in ies $6 \Delta$ cells (right) across all protein-coding and non-coding genes of $S$. pombe (from - $0.5 \mathrm{~kb}$ of the TSS to $+0.5 \mathrm{~kb}$ of the TES; $n=7,106)$. The heatmaps were sorted in descending order of les 6 enrichment. TES, transcription termination site; TSS, transcription start site. c Average gene profiles $(n=7,106)$ for les6 binding and histone H3 changes in ies6 $\Delta$ cells. $\mathbf{d}$ Venn diagram showing overlap of genes with high les 6 binding and those with high histone $\mathrm{H} 3$ increases in ies $6 \Delta$ cells, as individually determined by $k$-means clustering $(k=2)$. $P$-values were calculated using the hypergeometric distribution. e ChIP-Seq profiles at a representative euchromatic region for: lec1-5×Flag; les6-5×Flag; histone $\mathrm{H} 3$

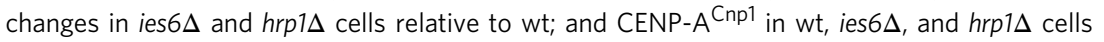

nucleosome ${ }^{18}$. In humans, RSF1 (remodeling and spacing factor 1) localizes to centromeres and promotes the stable association of CENP-A with centromeres ${ }^{19}$. A recent study showed that RSF1 can stimulate histone exchange accompanied by CENP-A deposition when artificially targeted to ectopically inserted $\alpha$ satellite DNA ${ }^{20}$. In addition to RSF1, the conserved ATP- dependent chromatin-remodeling factor, CHD1 (chromodomain helicase DNA binding protein 1), was found to stimulate the deposition of CENP-A ${ }^{\mathrm{Cnp} 1}$ at centromeres in human and fission yeast $^{21,22}$. In these studies, however, RSF1 and CHD1 were found to be only partially involved in the deposition of CENP-A at the centromeres. This raises the possibility that other chromatin- 
remodeling factor(s) are also responsible for regulating CENP-A chromatin assembly at regional centromeres. Also, it remains unclear whether the critical role played by chromatin-remodeling factors at the centromeres is related to the exchange of histone $\mathrm{H} 3$ with CENP-A.

To address these questions, here we systematically analyze the roles of the ATP-dependent chromatin-remodeling factors in the centromeric chromatin assembly of fission yeast. We find that the Ino80 complex plays an important role in CENP-A ${ }^{\mathrm{Cnp1}}$ chromatin assembly at the fission yeast centromeres in a redundant manner with $\mathrm{Chd} 1^{\mathrm{Hrpl}}$, and the role of the Ino80 complex is linked to removal of histone $\mathrm{H} 3$-containing nucleosomes. Consistent with a direct role in CENP-A ${ }^{\text {Cnp1 }}$ chromatin assembly, the Ino80 complex is associated with centromeric regions in a CENP$\mathrm{A}^{\mathrm{Cnp} 1}$-dependent manner and when tethered to a noncentromeric DNA inserted in an endogenous centromere promotes spreading of CENP-A ${ }^{\text {Cnp1 }}$ chromatin into the noncentromeric DNA. Thus, CENP-A ${ }^{\text {Cnp1 }}$ chromatin utilizes the Ino80 complex together with Chd $1^{\mathrm{Hrp} 1}$ to mediate replacement of histone $\mathrm{H} 3$ with CENP-A ${ }^{\mathrm{Cnp} 1}$ at regional centromeres of fission yeast.

\section{Results}

The Ino80 complex promotes $\mathrm{CENP}-\mathrm{A}^{\mathrm{Cnp1}}$ chromatin assembly. To identify the ATP-dependent chromatin-remodeling factors involved in the replacement of histone $\mathrm{H} 3$ with CENP-A $\mathrm{A}^{\mathrm{Cnp} 1}$ at centromeres, we first examined whether any of the fission yeast chromatin-remodeling factors could affect CENP-A ${ }^{\text {Cnp1 }}$ chromatin at centromeres. The silencing of $\mathrm{urat}^{+}$ inserted into a central core region of centromere 1 (cnt1:ura $\left.4^{+}\right)$ was previously used to assess the integrity of CENP-A $\mathrm{A}^{\mathrm{Cn} 1}$ chromatin in fission yeast ${ }^{23,24}$. Utilizing this assay, we screened a pool of fission yeast strains carrying mutations in chromatin remodeling factors for defective central core silencing. As previously shown ${ }^{21,25}$, the loss of Chd1 ${ }^{\mathrm{Hrpl}}$ disrupted central core silencing (Supplementary Fig. 1a, b). In addition, we found that the loss of Ies2, a non-essential component of the Ino80 chromatin-remodeling complex, also disrupted silencing (Supplementary Fig. 1b). Other viable mutants of the Ino80 complex, including iec $1 \Delta$ and ies $6 \Delta$, were also defective in silencing (Fig. 1a). Consistent with these findings, chromatin immunoprecipitation (ChIP) analyses showed that mutations of the Ino80 complex, most notably iec $1 \Delta$ and ies $6 \Delta$, reduced the occupancy of CENP-A ${ }^{\text {Cnp1 }}$ in the central core region (Fig. $1 \mathrm{~b}$ and Supplementary Fig. 1c). Using ChIP combined with high-throughput sequencing analysis (ChIP-Seq), we observed that the CENP-A ${ }^{\text {Cnp1 }}$ levels at all centromeres were majorly reduced in ies $6 \Delta$ cells and to a smaller degree in hrp $1 \Delta$ cells (Fig. 1c and Supplementary Fig. 2). Defects in centromere function often increase the sensitivity of cells to microtubuledestabilizing drugs, such as thiabendazole (TBZ), and increase the rates of chromosome loss 24,26 . We found that iec1 $\Delta$ and ies6 $\Delta$ induce TBZ sensitivity and dramatically increase the loss of a non-essential minichromosome (Fig. 1d, e), indicating that the impaired assembly of CENP-A ${ }^{\mathrm{Cnp} 1}$ chromatin in cells harboring defective Ino80 complex can disrupt normal centromere function.

The Ino80 complex evicts histone $\mathbf{H} 3$ from chromatin. Next, we used ChIP-Seq to determine whether the Ino80 complex acts directly at centromeres. This analysis revealed that the Iec1 and Ies6 subunits were widely associated with central kinetochore domains (i.e., cnt and the inner parts of $i m r$ ), as well as with specific regions of pericentromeric heterochromatin (otr) and proximal euchromatin (Fig. 2a and Supplementary Fig. 3). Notably, we found that localization of the Ino80 subunits in these regions coincided with the increased levels of histone $\mathrm{H} 3$ in ies6 $\Delta$ and $h r p 1 \Delta$ cells (Fig. 2a and Supplementary Fig. 3), directly implicating the Ino80 complex and $\mathrm{Chd1} 1^{\mathrm{Hrpl}}$ in the removal of histone $\mathrm{H} 3$-containing nucleosomes ${ }^{27}$. We observed that low levels of CENP-A ${ }^{\mathrm{Cnp} 1}$ incorporation were frequently observed at or near the centromere-proximal regions (pericentromeric heterochromatin and proximal euchromatin) where the Ino80 complex and Chd $1^{\text {Hrpl } 1}$ act to remove histone $\mathrm{H} 3$ (Fig. 2a and Supplementary Fig. 3). This suggests that the active removal of histone $\mathrm{H} 3$-containing nucleosomes by chromatin-remodeling factors predisposes these regions to incorporate CENP-A Cnp1 nucleosomes. The weak enrichment of CENP-A ${ }^{\mathrm{Cnp} 1}$ at these centromere-proximal regions was impaired in $h r p 1 \Delta$ cells, but not in ies6 $\Delta$ cells (Fig. 2a and Supplementary Fig. 3). This indicates that the Ino80 complex acts primarily to remove histone H3-containing nucleosomes, which leads to the assembly of CENP-A ${ }^{\text {Cnpl } 1}$ in the central domain. However, unlike Chd1 ${ }^{\mathrm{Hrp} 1}$, the Ino80 complex outside the central domain may guide only futile nucleosome exchanges, such as the reciprocal exchange of a histone $\mathrm{H} 3$-containing nucleosome with a CENP-A $\mathrm{Anp}^{\mathrm{C}}$ nucleosome, or the exchange of one histone H3-containing nucleosome with another. In contrast to ies6 $\Delta$, the mis18-262 mutation, which disrupts kinetochore assembly ${ }^{28}$, was associated with reduced CENP-A ${ }^{\text {Cnp1 }}$ nucleosome assembly at both central domains and centromere-proximal regions (Supplementary Fig. 4). This suggests that the Ino80 complex is not involved in the kinetochoredependent deposition or maintenance of CENP-A ${ }^{C n p 1}$. To test this further, we analyzed the effect of ies $6 \Delta$ on the centromeric localization of Scm3, a CENP-A $\mathrm{Cnp}^{\mathrm{C}}$-specific histone chaperone orthologous to human HJURP ${ }^{29-33}$. The association of $\mathrm{Scm} 3$ with centromeres is known to require various kinetochore proteins, including Sim4, Mis6, Mis16, and Mis18, but not CENP-A ${ }^{\text {Cnp1 }}$ itself $^{29}$. We found that binding of Scm 3 to the centromere is not significantly impaired in ies6 $\Delta$ cells (Supplementary Fig. 5), indicating that the CENP-A $\mathrm{A}^{\mathrm{Cn} 1}$ deposition pathway is likely to be intact in ies $6 \Delta$ cells.

As in budding yeast, the Ino80 subunits of fission yeast are also associated with other genomic loci, especially near the start and end of genes ${ }^{34,35}$ (Fig. 2b, c and Supplementary Fig. 6a, b). This non-centromeric localization of the Ino80 complex allowed us to examine whether the Ino80 complex acts to remove histone H3containing nucleosomes regardless of CENP-A ${ }^{\mathrm{Cnp} 1}$ chromatin assembly. Indeed, and consistent with the notion that the Ino80 complex plays a direct role in histone $\mathrm{H} 3 \mathrm{removal}^{27}, 36$, we found that the levels of histone $\mathrm{H} 3$ in ies $6 \Delta$ cells were increased in regions that are normally bound by Ies6 (Fig. 2b, c). Corroborating this, a Venn diagram analysis revealed that there is a significant overlap between genes showing high levels of Ies6 binding and those exhibiting robust increases in $\mathrm{H} 3$ levels in ies $6 \Delta$ cells (Fig. $2 \mathrm{~d}$ ). We found that $\mathrm{Chd1} 1^{\mathrm{Hrpl}}$ also promotes histone $\mathrm{H} 3$ removal at non-centromeric regions similar to those targeted by the Ino80 complex (near the start and end of genes), but it plays a weaker role in evicting $\mathrm{H} 3$ from euchromatin (Fig. 2e and Supplementary Fig. 7a, b). We also confirmed that the removal of histone $\mathrm{H} 3$ by the Ino80 complex at euchromatin is not tied to the assembly of CENP-A $\mathrm{A}^{\mathrm{Cnp} 1}$ (Fig. $2 \mathrm{e}$ and Supplementary Fig. 7c).

In budding yeast, the Ino80 complex acts to remove the histone $\mathrm{H} 2 \mathrm{~A}$ variant, H2A. $\mathrm{Z}^{\mathrm{Htz} 1}$, from chromatin ${ }^{16,34}$. Since H2A.Z $\mathrm{Z}^{\mathrm{Pht} 1}$ is depleted from fission yeast centromeres ${ }^{37,38}$, we questioned whether the defective CENP-A ${ }^{\text {Cnp1 }}$ chromatin assembly of ies6 $\Delta$ cells could be caused by the insufficient removal of H2A. $Z^{\text {Pht1 }}$, rather than $\mathrm{H} 3$, from the centromeres. In sharp contrast to the prominent increase in $\mathrm{H} 3$ occupancy seen in ies $6 \Delta$ cells, however, we found that ies $6 \Delta$ does not cause a significant increase in $\mathrm{H} 2 \mathrm{~A}$. $\mathrm{Z}^{\mathrm{Pht1}}$ occupancy at the centromeres (Supplementary Fig. 8a, b). 
a

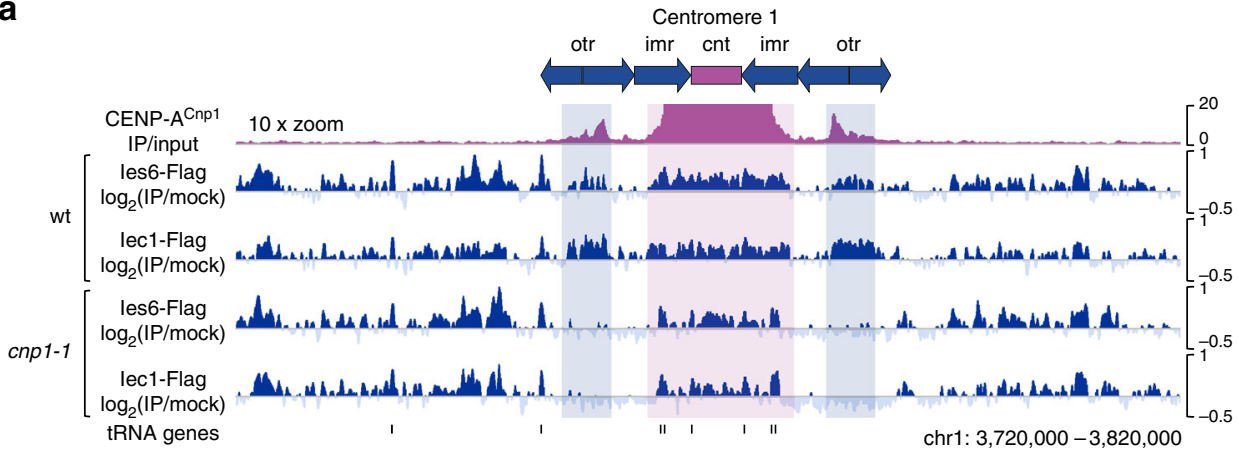

b $\stackrel{\text { wt }}{\text { neocen }}_{\text {cen1s tel1R-neocen }}^{\text {cen1 }}$

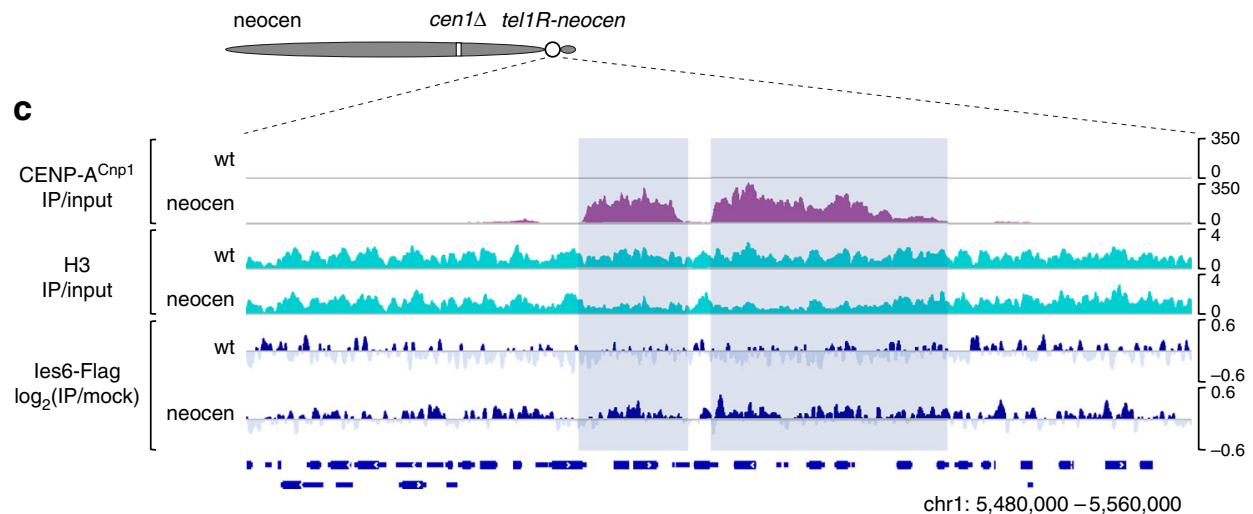

Fig. 3 The Ino80 complex is recruited to centromeres through CENP-A ${ }^{C n p 1}$ chromatin. a Magnified ( $\left.\times 10\right)$ views of CENP-A ${ }^{C n p 1}$ in wt cells and ChIP-Seq profiles of les6-5×Flag and lec1-5×Flag in wt and cnp1-1 cells at centromere 1. For Flag ChIP-Seq, wt and cnp1-1 cells were incubated at $36^{\circ} \mathrm{C}$ (restrictive temperature for cnp1-1) for $3 \mathrm{~h}$ after being grown at $25^{\circ} \mathrm{C}$ (permissive temperature for cnp1-1). The magenta and blue vertical shadings indicate the major CENP-A $A^{C n p 1}$ peaks in the central domain and the minor CENP-A ${ }^{C n p 1}$ peaks in the heterochromatin, respectively. $\mathbf{b} A$ schematic showing the positions of active centromeres (white circles) in chromosome 1 of the wt and neocentromere (neocen) strains. The neocentromere strain carries a neocentromere (tellR-neocen) assembled at a subtelomeric region, but lacks the endogenous centromere 1 (cen $1 \Delta$; white box). c Genome browser view of the tellR-neocen locus showing the ChIP-Seq profiles of CENP-A ${ }^{\mathrm{Cnp} 1}$, histone $\mathrm{H} 3$, and les6-5×Flag in the wt and neocentromere strains. The blue vertical shadings indicate the major CENP-A ${ }^{C n p 1}$ peaks in the neocentromere

Moreover, loss of H2A.Z $\mathrm{Z}^{\mathrm{Pht1}}$ did not rescue the defective central core silencing of ies $2 \Delta$ cells (Supplementary Fig. 8c), indicating that this silencing defect is not caused by H2A.Z $\mathrm{Z}^{\text {Pht1 }}$. As previously reported in budding yeast ${ }^{16}, 34$, we observed that H2A.Z ${ }^{\text {Pht1 }}$ accumulates at both gene promoters and coding regions in ies6 6 cells (Supplementary Fig. $8 \mathrm{~d}$, e). We thus conclude that the Ino80 complex affects CENP- $\mathrm{A}^{\text {Cnp1 }}$ chromatin in a manner independent of its role in restricting the localization of H2A.Z $Z^{\text {Pht1 }}$.

The action of the Ino80 complex at gene promoters prompted us to question whether the observed effect of ies $6 \Delta$ on CENP$\mathrm{A}^{\mathrm{Cnp} 1}$ chromatin could be indirectly caused by alterations in the expression levels of specific factors that regulate CENP-A ${ }^{\text {Cnp1 }}$ chromatin assembly. To exclude this possibility, we used mRNASeq analysis to perform gene expression profiling of ies $6 \Delta$ and wild-type (wt) cells. Consistent with the notion that the Ino80 complex plays a role in transcription, we identified hundreds of genes whose mRNA levels are significantly down- or up-regulated in ies6 $\Delta$ cells (Supplementary Table 1). However, among the genes whose functions are known to be important for CENP$\mathrm{A}^{\mathrm{Cnp1}}$ chromatin assembly, only $\operatorname{cnp} 3^{+}$, which encodes the

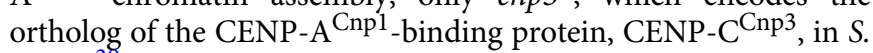
pombe $e^{39}$, showed significantly reduced expression in ies $6 \Delta$ cells (Supplementary Table 1). A noticeable observation that we made to solve this issue was that under a suboptimal growth condition (at $25^{\circ} \mathrm{C}$ in minimal EMM), ies6 $\Delta$ does not downregulate $c n p 3^{+}$ expression (Supplementary Fig. 9a), but ies6 $\Delta$ cells grown under this growth condition still show a significantly reduced level of CENP-A ${ }^{\text {Cnp1 } 1}$ at the centromere (Supplementary Fig. 9b). Therefore, we conclude that the Ino80 complex regulates CENP-A ${ }^{\text {Cnp } 1}$ chromatin regardless of its involvement in regulating $c n p 3^{+}$ expression.

The Ino80 complex functions redundantly with $\mathrm{Chd1}^{\mathrm{Hrp}}{ }^{\mathrm{1}}$. The weaker effect of $h r p 1 \Delta$ on CENP-A ${ }^{C n p 1}$ chromatin suggests that Chd $1{ }^{\text {Hrpl }}$ may complement the function of the Ino80 complex. We were unable to generate ies6 $h r p 1 \Delta$ cells, suggesting that ies $6 \Delta$ and $h r p 1 \Delta$ are synthetically lethal. The ies $2 \Delta$ hrp $1 \Delta$ double-mutant cells were viable, and showed larger defects in central core silencing than those observed in each of the single mutants (Supplementary Fig. 10a). The combination of $h r p 1 \Delta$ and a mutation affecting the RSC (remodeling the structure of chromatin) chromatin-remodeling comple ${ }^{40}$ did not produce this additive defect in silencing (Supplementary Fig. 10b, c), nor did the RSC mutant $(r s c 1 \Delta)$ cause any silencing defect when combined with ies2 $\Delta$ (Supplementary Fig. 10d). To further investigate the functional redundancy of Ino80 and $\mathrm{Chd1}^{\mathrm{Hrp1}}$, we generated strains carrying mutations in the essential catalytic subunit, Ino80, which show a cold-sensitive central core silencing defect (see Methods for more detail). When we combined the silencing-defective ino80-11 with $h r p 1 \Delta$, we observed a 
cumulative loss of both silencing and cell viability (Supplementary Fig. 11a). Consistent with this, we found that the combination of ino80-11 with $h r p 1 \Delta$ produces a more prominent loss of CENP-A ${ }^{\text {Cnp1 }}$ at the centromere than either of the single mutations (Supplementary Fig. 11b). The level of histone H3 occupancy at the centromere was also increased most significantly in the double mutant (ino80-11 hrp1s) compared to the wt (Supplementary Fig. 11c). These data indicate that Ino80 and
Chd $1{ }^{\mathrm{Hrp} 1}$ share a role in promoting the replacement of histone H3 with CENP-A Cnp1.

CENP-A ${ }^{\text {Cnp1 }}$ chromatin actively recruits the Ino80 complex. The association of the Ino80 complex with specific non-centromeric regions, such as gene promoters, prompted us to ask whether the centromeric localization of the Ino80 complex is mediated by CENP-A ${ }^{\text {Cnpl }}$ chromatin or by specific features of a

cnt1:bighyg

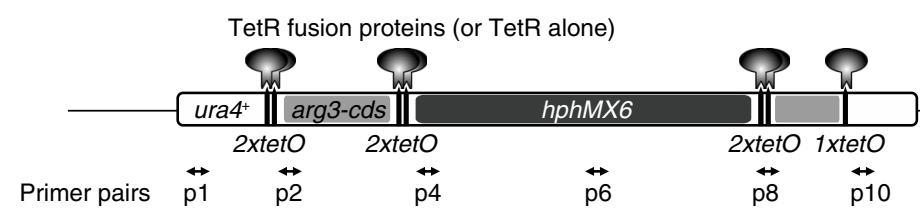

b

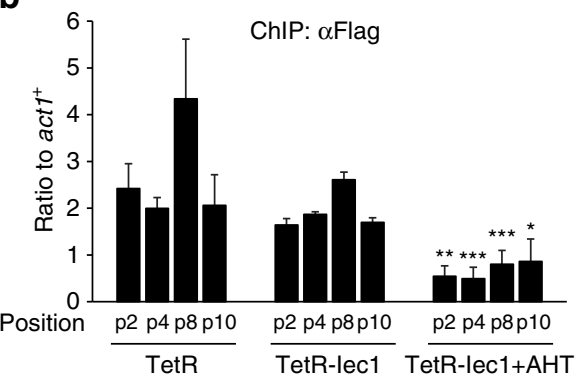

C
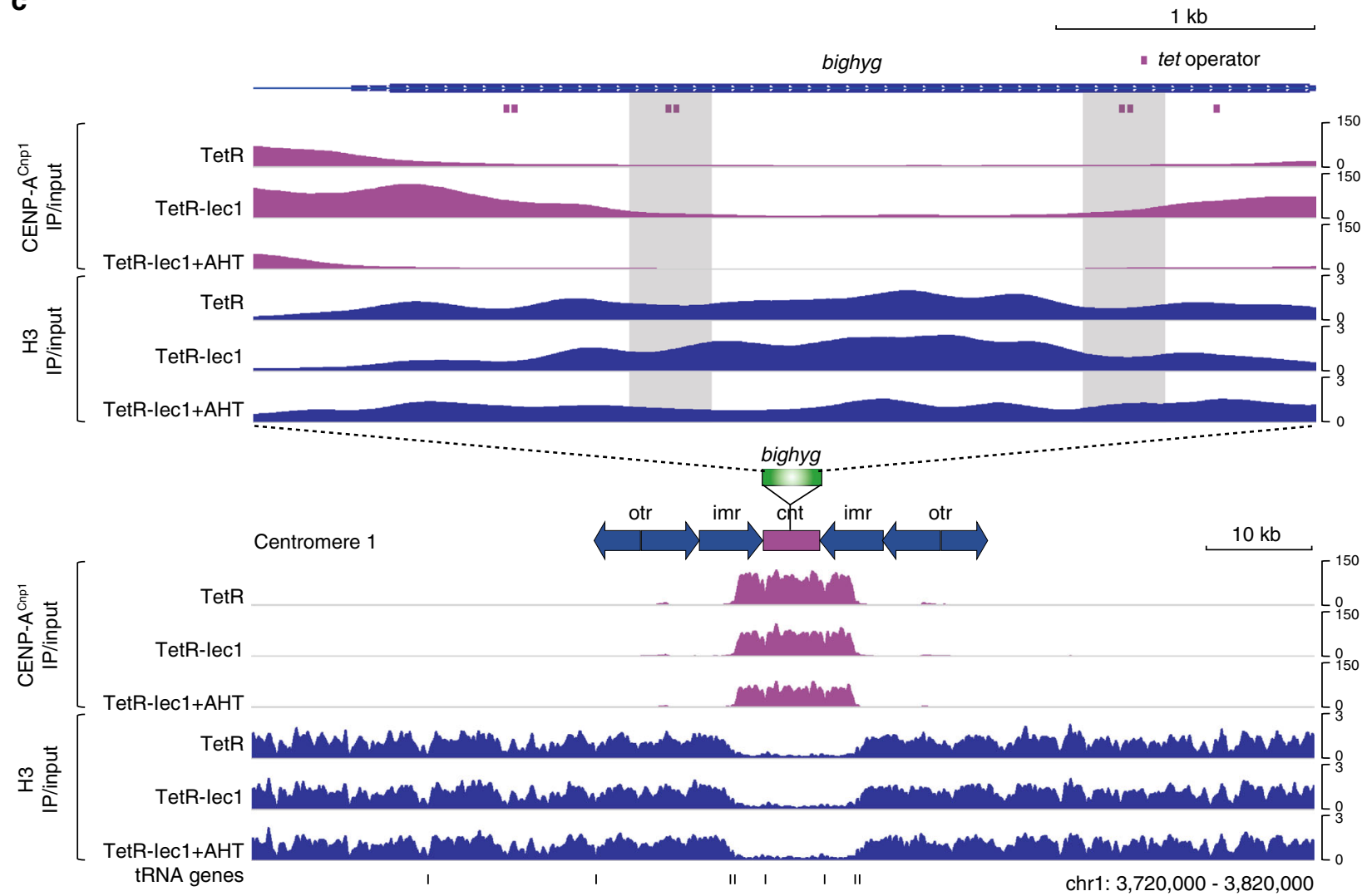

d

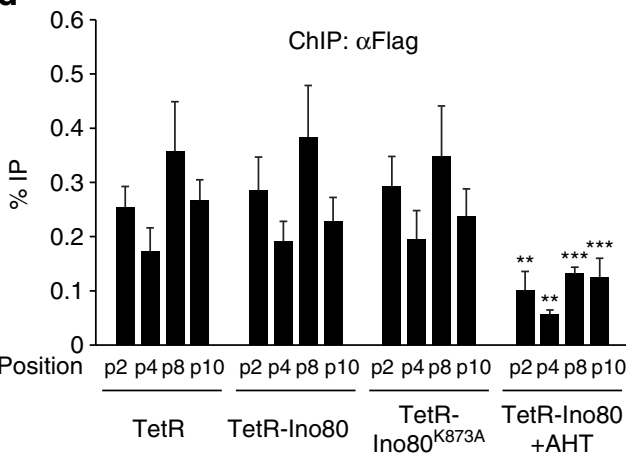

e

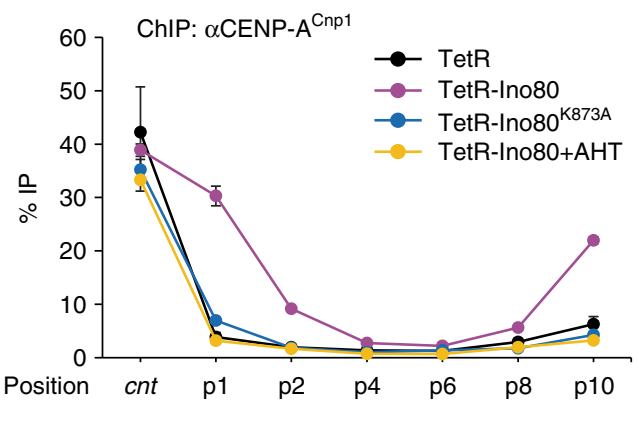


centromeric DNA sequences. To address this question, we examined whether CENP-A ${ }^{\mathrm{Cnp} 1}$ is required for the centromeric localization of the Ino80 complex. We found that in cells with defective CENP-A ${ }^{\text {Cnp1 }}$ (cnp1-1), the overall levels of Iec1 and Ies6 bound to the central domain (magenta vertical shading) were diminished and became constrained to more specific regions, including the transfer RNA genes (Fig. 3a). Interestingly, the association of the Ino80 complex with pericentromeric heterochromatin regions (otr) harboring low levels of CENP-A ${ }^{\text {Cnp1 }}$ (blue vertical shading) was completely abolished in cnp1-1 cells (Fig. 3a). These results indicate that the widespread associations of the Ino80 complex with CENP-A ${ }^{\text {Cnp1 }}$-enriched domains occur through CENP-A ${ }^{\mathrm{Cnp} 1}$. However, we cannot rule out the possibility that DNA sequence features may also play some role ${ }^{41}$. We

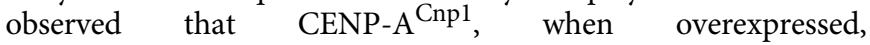
co-immunoprecipitates with Ies6, which raises the possibility that CENP-A ${ }^{\text {Cnp1 }}$ may have an interaction with the Ino80 complex (Supplementary Fig. 12). To further evaluate the significance of CENP-A ${ }^{\text {Cnp1 }}$ chromatin or DNA sequence cues in the centromeric localization of the Ino80 complex, we utilized a neocentromere strain that lacks the endogenous centromere of chromosome 1 and carries an ectopic centromere assembled at a non-centromeric region near the right telomere of chromosome 1 (tel1R-neocen) without any DNA sequence change ${ }^{42}$ (Fig. 3b). We found that Ies6 is widely associated with the tel1R-neocen locus in the neocentromere strain but not in the wt strain (Fig. 3c). Thus, CENP-A ${ }^{\text {Cnp1 }}$ chromatin, not the DNA sequence, majorly contributes to localizing the Ino80 complex to the neocentromere.

Tethered Ino80 subunits promote spreading of CENP-A ${ }^{\text {Cnp1 }}$. A large non-centromeric DNA placed in an endogenous centromere was previously shown to act as a poor substrate for the spreading of CENP-A ${ }^{\text {Cnp1 }}$ chromatin ${ }^{43}$. We used this to more directly address the function of the Ino80 complex in CENP$\mathrm{A}^{\mathrm{Cnp} 1}$ chromatin assembly. We tethered a TetR- $2 \times$ Flag-Iec1 (TetR-Iec1) fusion to tet $O$ sites in a $4.5 \mathrm{~kb}$ non-centromeric DNA (bighyg) sequence inserted at centromere 1, and examined whether tethered TetR-Iec1 alters CENP-A ${ }^{\text {Cnpl }}$ chromatin assembly at bighyg (Fig. 4a). We used TetR-2×Flag alone (TetR) and TetRIec1 released from bighyg by anhydrotetracycline (AHT) treatment as negative controls. We used ChIP analysis to confirm that TetR and TetR-Iecl were enriched at tet $O$ insertions and that TetR-Iec1 was released after the addition of AHT (Fig. 4b). A subsequent ChIP-Seq analysis showed that tethered TetR-Iec1 triggered the spread of CENP-A ${ }^{\text {Cnp1 }}$ chromatin into bighyg concurrent with a decline in histone $\mathrm{H} 3$ occupancy (Fig. 4c, compare TetR-Iec1 with TetR or TetR-Iec1+AHT). Tethering of TetR-Iec1 to more central regions of bighyg did not cause such a robust increase in CENP-A ${ }^{\text {Cnp1 }}$ chromatin assembly (gray vertical shadings in Fig. 4c). However, TetR-Iecl tethered to these regions causes a more notable local histone $\mathrm{H} 3$ depletion relative to surrounding regions than did tethered TetR (gray vertical shadings in Fig. 4c), which is consistent with the notion that
TetR-Iec1 induces the removal of histone H3-containing nucleosomes around the tethered sites. To determine whether the effect of tethered TetR-Iecl reflects the underlying catalytic activity of the Ino80 complex, we ectopically expressed TetRIno80 or TetR-Ino8 $00^{\mathrm{K} 873 \mathrm{~A}}$, which has a mutation in its conserved ATP-binding pocket ${ }^{44,45}$. We observed similar enrichments for TetR, TetR-Ino80, and TetR-Ino80 ${ }^{\mathrm{K} 873 \mathrm{~A}}$ at tet $O$ insertions, and TetR-Ino80 was successfully released after the addition of AHT (Fig. 4d). However, tethered TetR-Ino80, but not TetRIno80 ${ }^{\mathrm{K} 873 \mathrm{~A}}$, caused the spreading of CENP-A $\mathrm{Cnp}^{\mathrm{C}}$ chromatin into bighyg (Fig. 4e, compare TetR-Ino80 and TetR-Ino80 ${ }^{\mathrm{K} 873 \mathrm{~A}}$ at $\mathrm{p} 1, \mathrm{p} 2$, and $\mathrm{p} 10$ ), clearly implicating the ATPase activity of Ino80 in CENP-A ${ }^{\text {Cnp1 }}$ chromatin assembly. Moreover, we found that tethered Ino80 subunits exert a stronger effect on CENP-A ${ }^{\mathrm{Cnp} 1}$ assembly when placed closer to the native centromere (Fig. 4c-e). This supports that the Ino80 complex needs to be in a kinetochore context to stimulate CENP-A Cnp1 chromatin assembly, which is consistent with the observation that CENP-A ${ }^{\text {Cnp1 } 1}$ does not accumulate at non-centromeric regions where the Ino80 complex acts to remove histone $\mathrm{H} 3$ (Fig. 2e and Supplementary Fig. 7c).

\section{Discussion}

This study shows that the Ino80 complex is a critical chromatin-remodeling factor required for CENP-A ${ }^{\mathrm{Cnp} 1}$ chromatin assembly at the centromeres of fission yeast. Chd 1 Hrp1, which was previously implicated in CENP-A ${ }^{\mathrm{Cnp} 1}$ chromatin assembly $^{21}$, plays a redundant role with the Ino80 complex. Our findings in fission yeast may explain the discrepancy between human and Drosophila regarding the involvement of CHD1 in CENP-A chromatin assembly 2 , 46 , as the function of CHD1 could be easily concealed by the redundant function of other chromatin-remodeling factors such as the Ino80 complex.

Our conclusion that CENP-A ${ }^{\mathrm{Cnp} 1}$ chromatin rather than specific DNA sequence is the major determinant for centromeric localization of the Ino80 complex is supported by two main observations. First, the binding of Ino80 subunits is detected across the CENP-A ${ }^{\mathrm{Cnp} 1}$ chromatin domains, and this widespread association is largely dependent on CENP-A $\mathrm{Anp}^{\mathrm{Cn} 1}$ (Fig. 3a). Second, Ies6 associates with the site of neocentromere formation only when the neocentromere is activated and CENP-A ${ }^{\text {Cnp1 }}$ chromatin is assembled on the DNA (Fig. 3c).

Previous studies showed that the Ino80 complex in budding yeast also associates with the centromeres ${ }^{47}$ and promotes pericentric chromatin integrity and centromere function ${ }^{18}$, but does not affect the assembly of CENP-A ${ }^{\mathrm{Cse} 4}$ nucleosomes at the centromeres ${ }^{18}$. A recent study uncovered a rather unexpected role for the budding yeast Ino80 complex in promoting the ectopic deposition of CENP-A ${ }^{\text {Cse } 4}$ at gene promoters ${ }^{48}$. We speculate that the requirement for the Ino80 complex in the assembly of CENP-A nucleosomes at the centromeres was lost in budding yeast during the evolutionary transition from regional to point centromeres ${ }^{49}$. In contrast, the connection of the Ino80 complex

Fig. 4 Tethered Ino80 subunits enhance spreading of CENP-A ${ }^{C n p 1}$ chromatin into a non-centromeric DNA. a A schematic of cnt1:bighyg showing the positions of the tet operators (tetO), the TetR fusion proteins (or TetR alone) in bighyg. The bighyg DNA consists of ura $^{+}$, arg $3^{+}$coding sequence ( $\arg 3-$ $c d s)$, and the hygromycin-resistance gene (hphMX6). The utilized primers pairs are indicated below. b ChIP analysis for Flag-tagged TetR, TetR-lec1, and TetR-lec1 in cells treated with AHT (TetR-lec1+AHT), as assessed using the indicated primers. TetR-lec1 binding was significantly reduced after the addition of $\mathrm{AHT}$, as determined by Student's $t$-tests $\left({ }^{\star} P \leq 0.05,{ }^{\star \star} P \leq 0.01\right.$, and ${ }^{\star \star \star} P \leq 0.001$ ). c Genome browser view of bighyg (top) and centromere 1 (bottom) showing the ChIP-Seq profiles of CENP-A ${ }^{\mathrm{Cnp1}}$ (magenta) and histone H3 (blue) in TetR-expressing cells, TetR-lec1-expressing cells, and TetR-lec1expressing cells treated with AHT (TetR-lec1+AHT). Gray vertical shadings indicate $\sim 400$ bp regions encompassing tet $\mathrm{O}$ inserted in the central part of bighyg. d ChIP analysis for Flag-tagged TetR, TetR-Ino80, TetR-Ino80K873A, and TetR-Ino80 in cells treated with AHT (TetR-Ino80+AHT), as assessed

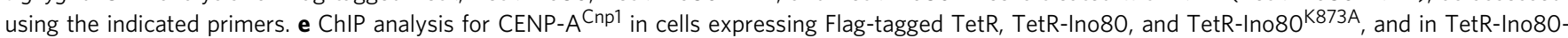
expressing cells treated with AHT (TetR-Ino80+AHT), as assessed using the indicated primers. Error bars in all ChIP data indicate the \pm s.d. for the biological replicates 
with centromeres ${ }^{18}$ and CENP-A ${ }^{48}$ was retained, allowing the Ino80 complex to exhibit divergent roles in budding yeast. The roles of the Ino80 complex in CENP-A chromatin assembly at regional versus point centromeres should be examined in the future to test this hypothesis.

As reported in budding yeast ${ }^{16,34}$, we herein show that the fission yeast Ino80 complex plays a prominent role in removing histone H2A.Z $\mathrm{Z}^{\text {Pht1 }}$ from chromatin (Supplementary Fig. 8d, e). However, we found that H2A.Z $Z^{\text {Pht1 }}$ does not significantly accumulate at the centromeres in ies6 $\Delta$ cells (Supplementary Fig. 8a, b) and the defective central core silencing in ies $2 \Delta$ cells is not rescued by the elimination of histone $\mathrm{H} 2 \mathrm{~A} . \mathrm{Z}^{\mathrm{Pht} 1}$ (Supplementary Fig. $8 \mathrm{c}$ ). These results indicate that the major function of the Ino80 complex in the assembly of CENP-A ${ }^{\text {Cnp1 }}$ chromatin at the centromeres is not likely to be the removal of histone H2A.Z $Z^{\text {Pht1 }}$. Instead, our analysis revealed that the localization of the Ino80 complex was tightly correlated with its role in histone $\mathrm{H} 3$ eviction rather than CENP-A Cnpl deposition (Fig. 2). Our findings thus support a model in which the Ino80 complex mainly acts at the centromeres to remove histone $\mathrm{H} 3$-containing nucleosomes as a prerequisite for CENP-A $\mathrm{A}^{\mathrm{Cnp} 1}$ deposition. We note that the Ino80 complex was also shown to induce full nucleosome turnover in budding yeast ${ }^{34}$. Kinetochore proteins, such as CENP-C $\mathrm{C}^{\mathrm{Cnp} 3}$, may stabilize CENP-A ${ }^{\mathrm{Cnp} 1}$ nucleosomes at centromeres ${ }^{50}$, thereby generating a chromatin context in which the Ino80 complex selectively evicts histone $\mathrm{H} 3$-containing nucleosomes. Extending our model, a recent study showed that the chromatin-remodeling factor, RSF1, can stimulate histone H3.3 exchange as well as CENP-A deposition in humans ${ }^{20}$. Based on the present and prior findings, we envision that the exchange of histone $\mathrm{H} 3$-containing nucleosomes with CENP-A nucleosomes by centromere-targeted chromatin-remodeling factors could be a conserved theme underpinning the epigenetic propagation of CENP-A chromatin at regional centromeres.

\footnotetext{
Methods

Strains, drugs and standard techniques. Standard genetic and molecular techniques were used. YES (Yeast Extract with Supplements) was used as a rich medium and PMG (Pombe Minimal Glutamate) or EMM (Edinburgh Minimal Medium) was used as a minimal medium. For the selective and non-selective media in the silencing assays, we used PMG supplemented with amino acids. For 5fluoroorotic acid (FOA) selection, we added FOA (final concentration $1 \mathrm{gl}^{-1}$ ) to PMG media. Where indicated, we added TBZ (final concentration $10 \mu \mathrm{g} \mathrm{ml}^{-1}$; Sigma) dissolved in dimethyl sulfoxide to the YES plates and AHT (final concentration $10 \mu \mathrm{M}$; Sigma) dissolved in DMSO to the YES or PMG media. We incubated all fission yeast cells at $30^{\circ} \mathrm{C}$ unless otherwise indicated. We used PCRbased gene targeting or genetic crosses followed by random spore analysis to create the deletion and Flag-tagged strains. We were able to infer the synthetic lethality of ies $6 \Delta h r p 1 \Delta$ by crossing ies $6 \Delta$ and $h r p 1 \Delta$ and performing a random spore analysis; we were unable to find any ies6 $\Delta::$ natMX6 hrp1 $1:$ kanMX6 $\left(\right.$ nat $^{\mathrm{R}}$ kan $\left.^{\mathrm{R}}\right)$ spores despite selecting over 300 ies6 $\Delta::$ natMX6 $\left(n a t^{\mathrm{R}}\right)$ spores. For more details regarding the yeast strains used in each experiment, see Supplementary Table 2.
}

ChIP-Seq and data analysis. Cells were fixed with $1 \%$ formaldehyde for $10 \mathrm{~min}$ (for cells grown at $36^{\circ} \mathrm{C}$ ), $15 \mathrm{~min}$ (for cells grown at $30^{\circ} \mathrm{C}$ ), or $40 \mathrm{~min}$ (for cells grown at $20^{\circ} \mathrm{C}$ ). ChIP-Seq required more cells than conventional ChIP, as we needed to ensure that there was sufficient immunoprecipitated DNA for high-throughput sequencing. We used $1.6 \times 10^{9}, 4 \times 10^{8}$, and $2.4-3.2 \times 10^{9}$ cells for the CENP-A ${ }^{\mathrm{Cnp} 1} \mathrm{ChIP}, \mathrm{H} 3 \mathrm{ChIP}$, and Flag ChIP experiments, respectively, increasing the amount of lysis buffers and antibodies accordingly. For the CENP-A ${ }^{\text {Cnp1 }}$ and H3 ChIP-Seq, we used $70 \mu \mathrm{l}$ of in-house rabbit anti-CENP-A ${ }^{\text {Cnp1 }}$ antiserum and $15 \mu \mathrm{l}$ of in-house rabbit anti-H3 antiserum, respectively. For the Flag ChIP-Seq experiments, we used $10 \mu \mathrm{l}$ of anti-Flag M2 antibody $\left(1 \mathrm{mg} \mathrm{ml}^{-1}\right.$, mouse monoclonal, F1804; Sigma) and included equal numbers of wt cells (lacking any tag) in parallel as controls. We performed the ChIP experiments for CENP$\mathrm{A}^{\mathrm{Cnp} 1}$ and $\mathrm{H} 3$ with only minor modifications to conventional ChIP protocols. We prepared the cell extracts by disrupting the cells in lysis buffer

(50 mM HEPES-KOH, pH 7.5, $140 \mathrm{mM} \mathrm{NaCl}, 1 \mathrm{mM}$ EDTA, 1\% Triton X-100, $0.1 \%$ sodium deoxycholate) using a standard bead-beating protocol. Following immunoprecipitation (IP), we washed the DNA-bound protein Aagarose beads and eluted the DNA in IP columns (SigmaPrep spin columns, Sigma) instead of microfuge tubes. As the external spike-in control for the H3 ChIP-Seq experiments, formaldehyde-fixed wild-type S. cerevisiae (W303a) cells were added to the $S$ pombe cells at a 1:8 ratio before cell disruption. For the Flag ChIP experiments, we used a modified lysis buffer (50 mM Tris- $\mathrm{HCl}, \mathrm{pH} 7.5,150 \mathrm{mM} \mathrm{NaCl}, 1 \mathrm{mM}$ EDTA, 1\% Triton X-100) and Tris-buffered saline (TBS) (20 mM Tris-HCl, pH 7.5, $150 \mathrm{mM} \mathrm{NaCl}$ )-based wash buffers (TBS and TBS with $0.05 \%$ Tween-20) to avoid denaturation of the M2 antibody by sodium deoxycholate. As the external spike-in control for the H2A. $Z^{\text {Pht1 }}-5 \times$ Flag ChIP-Seq experiments, formaldehyde-fixed $S$. cerevisiae cells expressing Sua7 $-5 \times$ Flag were added to $S$. pombe cells at a 1:9 ratio before cell disruption. We recovered the de-crosslinked DNA using a Qiagen PCR purification kit. We constructed the ChIP-Seq libraries with 5 to $10 \mathrm{ng}$ of input DNA or 1 to $10 \mathrm{ng}$ of immunoprecipitated DNA, using a ChIP-Seq kit (NEXTflexChIP-Seq kit, Bioo Scientific) according to the manufacturer's protocol. For multiplexed libraries, we sequenced $50 \mathrm{nt}$ single-end reads on an Illumina HiSeq 2500 (Theragen and Macrogen). After removing the adapter sequences, we aligned the processed reads to the $S$. pombe ASM294v2 genome assembly using Novoalign. We randomly assigned single locations to reads that mapped to multiple locations. For reads obtained from duplicate samples, we confirmed their experimental reproducibility using the bamCorrelate tool from the deepTools package ${ }^{51}$. For quantitative analysis of the data obtained from the $\mathrm{H} 3$ and $\mathrm{H} 2 \mathrm{~A} . \mathrm{Z}^{\mathrm{Pht1}} \mathrm{ChIP}-\mathrm{Seq}$ experiments, we calculated the IP/input ratio for each ChIP normalized to that of the spike-in control, which in principle should be constant in all ChIP samples. The normalized ratio of IP/input $\left(\mathrm{E}^{\mathrm{IP}}\right)$ was derived as:

$\mathrm{E}^{\mathrm{IP}}=(\mathrm{read}$ count of IP mapped to $S$. pombe genome/read count of input mapped to $S$. pombe genome)/(read count of IP mapped to $S$. cerevisiae genome/read count of input mapped to $S$. cerevisiae genome).

We computed the fold change of IP versus the input using the

deepToolsbamCompare tool. The normalization factor $(N)$ for the IP/input in each ChIP-Seq experiment was calculated as:

$N \times$ read count of IP/read count of input $=\mathrm{E}^{\mathrm{IP}}: 1$.

When we directly computed the fold change of IP in the mutant versus wild type, the normalization factor $(N)$ for $\mathrm{IP}^{\text {mutant }} / \mathrm{IP}^{\mathrm{wt}}$ was calculated as:

$N \times$ read count of $\mathrm{IP}^{\text {mutant }} / \mathrm{read}$ count of $\mathrm{IP}^{\mathrm{wt}}=\mathrm{E}^{\mathrm{IP}}$ of mutant $/ \mathrm{E}^{\mathrm{IP}}$ of wt.

The normalization of ChIP-Seq for CENP-A ${ }^{\text {Cnp1 }}$, Iec1-5 $\times$ Flag and Ies6-5×Flag was performed using the signal extraction scaling method. For visualization of the normalized data, we used the integrative genome viewer. We used the deepTools computeMatrix tool to perform an average gene (metagene) analysis and the profiler tool from deepTools to visualize the average gene profiles. We generated heatmaps using the deepToolsHeatmapper. We performed all further downstream bioinformatic analyses of the ChIP-Seq data using the R software package.

ChIP analysis. We performed the Fast ChIP protocol ${ }^{52}$ using $20 \mu \mathrm{l}$ of anti-CENP $\mathrm{A}^{\mathrm{Cnp} 1}$ antibody (rabbit polyclonal; in-house), $8 \mu \mathrm{l}$ of anti-H3 antibody (rabbit polyclonal; in-house), $2 \mu \mathrm{l}$ of anti-Flag M2 antibody $\left(1 \mathrm{mg} \mathrm{ml}^{-1}\right.$, mouse monoclonal, F1804; Sigma), and $2 \mu \mathrm{l}$ of anti-GFP tag antibody $\left(2 \mathrm{mg} \mathrm{ml}^{-1}\right.$, rabbit polyclonal, A-11122; Thermo Fisher). We used the Bio-Rad CFX96 system to analyze the input or IP DNA by quantitative PCR. See Supplementary Table 3 for the primer sequences.

mRNA-Seq analysis. We extracted total RNAs and isolated mRNAs from wt and ies $6 \Delta$ cells using poly(A)beads (NEXTflex). The stranded libraries were generated from biological replicates of each strain using NEXTflex Rapid Directional RNASeq Kit according to the manufacturer's manual and sequenced as single end. The sequence reads were aligned using STAR aligner ${ }^{53}$. Cufflinks tools were used to assemble the transcripts, to calculate their abundance in FPKM, and to determine list of genes that are differentially expressed in ies $6 \Delta$ cells compared to wt. Genes whose expressions were increased or decreased more than twofold in ies $6 \Delta$ cells relative to wt with $q$-value (false discovery rate-adjusted $p$-value) of ${ }^{<} 0.05$ were defined as significantly upregulated or downregulated genes, respectively (Supplementary Table 1).

Generation of silencing-defective alleles of ino $\mathbf{8 0}^{+}$. To identify mutant alleles of ino $80^{+}$, we used a Gene Morph II random mutagenesis kit (Stratagene) to mutagenize a DNA fragment containing the terminal $1.4 \mathrm{~kb}$ of the $i n o 80^{+}$open reading frame (ORF), which covered a significant portion of the catalytic domain. We then used fusion PCR to fuse the mutagenized fragments to a $\mathrm{kan}^{R}$ marker gene and sequences downstream of the ino80 $0^{+}$ORF. We selected transformants on G418containing plates by screening for slow growth at $35.5^{\circ} \mathrm{C}$. Then, we further screened these mutants for defective silencing of cnt1:ura4 ${ }^{+}$at different temperatures $\left(25,30,32.5,35.5^{\circ} \mathrm{C}\right)$. This technique allowed us to isolate several coldsensitive silencing-defective mutant alleles, including ino80-11. We identified the respective causative mutations in selected each strain using standard Sanger sequencing.

Minichromosome loss assay. We used genetic crossing to introduce a non-essential minichromosome (chromosome 16 (Ch16)). Ch16 bears the S. cerevisiae LEU2 gene, which allows it to complement leu1-32. We cultured wt and mutant cells containing Ch16 in minimal medium lacking leucine (PMG-Leu) to maintain the minichromosome. We plated these cells on non-selective (YES) plates with or without allowing them to divide 10 to 12 times in non-selective medium 
(YES). We then replica-plated the resulting colonies onto selective medium lacking leucine (PMG-Leu) and counted those that could and could not grow on selective medium (PMG-Leu). We calculated the rate of minichromosome loss using the following formula: loss rate per division $(\%)=100 \times\left(1-(F / I)^{1 / N}\right)$, where $F$ is the final percentage of minichromosome-containing cells, $I$ is the initial percentage of minichromosome-containing cells, and $N$ is the number of generations ${ }^{54}$. We tested three independent colonies for each strain. Data are presented as the mean loss rate \pm s.d. (error bars).

Co-immunoprecipitation. Cell pellets from log-phase cultures were resuspended in NP40 lysis buffer (1\% NP40, $200 \mathrm{mM} \mathrm{NaCl}, 2 \mathrm{mM}$ EDTA, $6 \mathrm{mM} \mathrm{Na}_{2}$ HPO4, $4 \mathrm{mM}$ $\mathrm{NaH}_{2} \mathrm{PO}_{4}, 2 \mathrm{mM}$ PMSF, protease inhibitors) and lysed by bead-beating. Extracts were immunoprecipitated with $25 \mu \mathrm{l}$ anti-Flag (M2)-agarose (Sigma). Immunoprecipitates were subjected to western blotting for the presence of Ies6-5 $\times$ Flag or CENP- $\mathrm{A}^{\mathrm{Cnp1}}$ using horseradish peroxidase-conjugated anti-Flag (M2) antibody (1:5,000, A8592; Sigma) or affinity-purified anti-CENP-A ${ }^{\text {Cnp1 }}$ antibody (1:1,000; in-house), respectively.

Generation of tethering strains. We generated the cnt1:bighyg strain based on the cnt1: $\mathrm{rra}^{+}$strain, which contains $\mathrm{ura}^{+}$at the $\mathrm{NcoI}$ site of the central core region in centromere $1^{23}$. First, we used PCR to attach $2 \times$ tetO or $1 \times$ tetO to each end of a $1 \mathrm{~kb}$ DNA fragment derived from the internal coding region of $\arg 3^{+}$. We then integrated the resulting PCR fragments into the middle of $\mathrm{ura}^{+}$and integrated the hygromycin-resistance gene ( $h p h M X 6)$, which is flanked by $2 \times t e t O$, into the center of the $\arg 3^{+}$insertion. Finally, we deleted the endogenous $u r a 4^{+}$and $\arg 3^{+}$genes of the resulting cnt1:bighyg strain. The $u r a 4^{+}$terminator sequence at the end of cnt1: bighyg was excluded from the analysis because it is also present at the end of the endogenous $\mathrm{ura}^{+}$locus. We generated the TetR-2×Flag-Iecl fusion strain by replacing the start codon (ATG) of the endogenous iec1 ${ }^{+}$gene with a DNA fragment encoding TetR- $2 \times$ Flag. We generated the TetR- $2 \times$ Flag strain without the Iecl fusion by fusing the $i e c 1^{+}$promoter and the TetR- $2 \times$ Flag sequence and integrating them into an ectopic $\mathrm{rra}^{+}$locus to replace the promoter and coding region of ura 4 ${ }^{+}$. For the Ino80-tethering experiments, we replaced the promoter and coding region of $\mathrm{ura}^{+}$with the thiamine-repressible $n m t 81$ promoter and a DNA sequence encoding TetR-2×Flag-Ino80, TetR- $2 \times$ Flag-Ino $80^{\mathrm{K} 873 \mathrm{~A}}$, or

TetR-2 $\times$ Flag. We induced expression of the fusion proteins by growing the strains in PMG medium lacking thiamine. We generated the TetR fusion gene-containing strains and cnt1:bighyg by genetic crosses and verified them using diagnostic PCR.

Data availability. The raw and processed sequencing data from this publication have been submitted to the NCBI Gene Expression Omnibus (GEO; http://www. ncbi.nlm.nih.gov/geo/) under accession number GSE99589. The authors declare that all the data supporting the findings of this study are available within the article and its Supplementary Information files and from the corresponding author on reasonable request.

Received: 15 November 2016 Accepted: 19 July 2017

Published online: 13 September 2017

\section{References}

1. Mendiburo, M. J., Padeken, J., Fulop, S., Schepers, A. \& Heun, P. Drosophila CENH3 is sufficient for centromere formation. Science 334, 686-690 (2011).

2. Allshire, R. C. \& Karpen, G. H. Epigenetic regulation of centromeric chromatin: old dogs, new tricks? Nat. Rev. Genet. 9, 923-937 (2008).

3. Black, B. E. \& Cleveland, D. W. Epigenetic centromere propagation and the nature of CENP-a nucleosomes. Cell 144, 471-479 (2011).

4. Chen, C. C. \& Mellone, B. G. Chromatin assembly: journey to the CENter of the chromosome. J. Cell Biol. 214, 13-24 (2016).

5. McKinley, K. L. \& Cheeseman, I. M. The molecular basis for centromere identity and function. Nat. Rev. Mol. Cell Biol. 17, 16-29 (2016).

6. Jansen, L. E., Black, B. E., Foltz, D. R. \& Cleveland, D. W. Propagation of centromeric chromatin requires exit from mitosis. J. Cell Biol. 176, 795-805 (2007).

7. Dunleavy, E. M., Almouzni, G. \& Karpen, G. H. H3.3 is deposited at centromeres in $\mathrm{S}$ phase as a placeholder for newly assembled CENP-A in $\mathrm{G}(1)$ phase. Nucleus 2, 146-157 (2011).

8. Takahashi, K. et al. A low copy number central sequence with strict symmetry and unusual chromatin structure in fission yeast centromere. Mol. Biol. Cell 3, 819-835 (1992).

9. Partridge, J. F., Borgstrom, B. \& Allshire, R. C. Distinct protein interaction domains and protein spreading in a complex centromere. Genes Dev. 14, 783-791 (2000)

10. Allshire, R. C. \& Ekwall, K. Epigenetic regulation of chromatin states in Schizosaccharomyces pombe. Cold Spring Harb. Perspect. Biol. 7, a018770 (2015).
11. Lando, D. et al. Quantitative single-molecule microscopy reveals that CENP-A (Cnp1) deposition occurs during G2 in fission yeast. Open Biol. 2, 120078 (2012).

12. Castillo, A. G. et al. Plasticity of fission yeast CENP-A chromatin driven by relative levels of histone $\mathrm{H} 3$ and H4. PLoS Genet. 3, e121 (2007).

13. Boeger, H., Griesenbeck, J., Strattan, J. S. \& Kornberg, R. D. Nucleosomes unfold completely at a transcriptionally active promoter. Mol. Cell 11, 1587-1598 (2003).

14. Clapier, C. R. \& Cairns, B. R. The biology of chromatin remodeling complexes. Annu. Rev. Biochem. 78, 273-304 (2009).

15. Mizuguchi, G. et al. ATP-driven exchange of histone H2AZ variant catalyzed by SWR1 chromatin remodeling complex. Science 303, 343-348 (2004).

16. Papamichos-Chronakis, M., Watanabe, S., Rando, O. J. \& Peterson, C. L. Global regulation of H2A.Z localization by the INO80 chromatin-remodeling enzyme is essential for genome integrity. Cell 144, 200-213 (2011).

17. Konev, A. Y. et al. CHD1 motor protein is required for deposition of histone variant H3.3 into chromatin in vivo. Science 317, 1087-1090 (2007).

18. Chambers, A. L. et al. The INO80 chromatin remodeling complex prevents polyploidy and maintains normal chromatin structure at centromeres. Genes Dev. 26, 2590-2603 (2012)

19. Perpelescu, M., Nozaki, N., Obuse, C., Yang, H. \& Yoda, K. Active establishment of centromeric CENP-A chromatin by RSF complex. J. Cell Biol. 185, 397-407 (2009).

20. Ohzeki, J. et al. KAT7/HBO1/MYST2 regulates CENP-A chromatin assembly by antagonizing Suv39h1-mediated centromere inactivation. Dev. Cell 37, 413-427 (2016).

21. Walfridsson, J. et al. The CHD remodeling factor Hrp1 stimulates CENP-A loading to centromeres. Nucleic Acids Res. 33, 2868-2879 (2005).

22. Okada, M., Okawa, K., Isobe, T. \& Fukagawa, T. CENP-H-containing complex facilitates centromere deposition of CENP-A in cooperation with FACT and CHD1. Mol. Biol. Cell 20, 3986-3995 (2009).

23. Allshire, R. C., Javerzat, J. P., Redhead, N. J. \& Cranston, G. Position effect variegation at fission yeast centromeres. Cell 76, 157-169 (1994).

24. Pidoux, A. L., Richardson, W. \& Allshire, R. C. Sim4: a novel fission yeast kinetochore protein required for centromeric silencing and chromosome segregation. J. Cell Biol. 161, 295-307 (2003).

25. Choi, E. S. et al. Identification of noncoding transcripts from within CENP-A chromatin at fission yeast centromeres. J. Biol. Chem. 286, 23600-23607 (2011).

26. Takahashi, K., Yamada, H. \& Yanagida, M. Fission yeast minichromosome loss mutants mis cause lethal aneuploidy and replication abnormality. Mol. Biol. Cell 5, 1145-1158 (1994).

27. Hogan, C. J. et al. Fission yeast Iec1-ino80-mediated nucleosome eviction regulates nucleotide and phosphate metabolism. Mol. Cell Biol. 30, 657-674 (2010).

28. Hayashi, T. et al. Mis16 and Mis18 are required for CENP-A loading and histone deacetylation at centromeres. Cell 118, 715-729 (2004).

29. Pidoux, A. L. et al. Fission yeast Scm3: a CENP-A receptor required for integrity of subkinetochore chromatin. Mol. Cell 33, 299-311 (2009).

30. Williams, J. S., Hayashi, T., Yanagida, M. \& Russell, P. Fission yeast Scm3 mediates stable assembly of Cnp1/CENP-A into centromeric chromatin. Mol. Cell 33, 287-298 (2009).

31. Dunleavy, E. M. et al. HJURP is a cell-cycle-dependent maintenance and deposition factor of CENP-A at centromeres. Cell 137, 485-497 (2009).

32. Foltz, D. R. et al. Centromere-specific assembly of CENP-a nucleosomes is mediated by HJURP. Cell 137, 472-484 (2009).

33. Sanchez-Pulido, L., Pidoux, A. L., Ponting, C. P. \& Allshire, R. C. Common ancestry of the CENP-A chaperones Scm3 and HJURP. Cell 137, 1173-1174 (2009).

34. Yen, K., Vinayachandran, V. \& Pugh, B. F. SWR-C and INO80 chromatin remodelers recognize nucleosome-free regions near +1 nucleosomes. Cell $\mathbf{1 5 4}$ 1246-1256 (2013).

35. Xue, Y. et al. The Ino80 complex prevents invasion of euchromatin into silent chromatin. Genes Dev. 29, 350-355 (2015).

36. Barbaric, S. et al. Redundancy of chromatin remodeling pathways for the induction of the yeast PHO5 promoter in vivo. J. Biol. Chem. 282, 27610-27621 (2007).

37. Buchanan, L. et al. The Schizosaccharomyces pombe JmjC-protein, Msc1, prevents H2A.Z localization in centromeric and subtelomeric chromatin domains. PLoS Genet. 5, e1000726 (2009).

38. Ogiyama, Y., Ohno, Y., Kubota, Y. \& Ishii, K. Epigenetically induced paucity of histone H2A.Z stabilizes fission-yeast ectopic centromeres. Nat. Struct. Mol. Biol. 20, 1397-1406 (2013).

39. Tanaka, K., Chang, H. L., Kagami, A. \& Watanabe, Y. CENP-C functions as a scaffold for effectors with essential kinetochore functions in mitosis and meiosis. Dev. Cell 17, 334-343 (2009).

40. Monahan, B. J. et al. Fission yeast SWI/SNF and RSC complexes show compositional and functional differences from budding yeast. Nat. Struct. Mol. Biol. 15, 873-880 (2008). 
41. Catania, S., Pidoux, A. L. \& Allshire, R. C. Sequence features and transcriptional stalling within centromere DNA promote establishment of CENP-A chromatin. PLoS Genet. 11, e1004986 (2015).

42. Ishii, K. et al. Heterochromatin integrity affects chromosome reorganization after centromere dysfunction. Science 321, 1088-1091 (2008).

43. Choi, E. S. et al. Factors that promote $\mathrm{H} 3$ chromatin integrity during transcription prevent promiscuous deposition of CENP-A(Cnp1) in fission yeast. PLoS Genet. 8, e1002985 (2012).

44. Shen, X., Mizuguchi, G., Hamiche, A. \& Wu, C. A chromatin remodelling complex involved in transcription and DNA processing. Nature 406, 541-544 (2000).

45. Neumann, F. R. et al. Targeted INO80 enhances subnuclear chromatin movement and ectopic homologous recombination. Genes Dev. 26, 369-383 (2012).

46. Podhraski, V. et al. CenH3/CID incorporation is not dependent on the chromatin assembly factor CHD1 in Drosophila. PLoS ONE 5, e10120 (2010).

47. Ogiwara, H., Enomoto, T. \& Seki, M. The INO80 chromatin remodeling complex functions in sister chromatid cohesion. Cell Cycle 6, 1090-1095 (2007).

48. Hildebrand, E. M. \& Biggins, S. Regulation of budding yeast CENP-A levels prevents misincorporation at promoter nucleosomes and transcriptional defects. PLoS Genet. 12, e1005930 (2016).

49. Malik, H. S. \& Henikoff, S. Major evolutionary transitions in centromere complexity. Cell 138, 1067-1082 (2009).

50. Falk, S. J. et al. Chromosomes. CENP-C reshapes and stabilizes CENP-A nucleosomes at the centromere. Science 348, 699-703 (2015).

51. Ramirez, F., Dundar, F., Diehl, S., Gruning, B. A. \& Manke, T. deepTools: a flexible platform for exploring deep-sequencing data. Nucleic Acids Res. 42, W187-W191 (2014)

52. Nelson, J. D., Denisenko, O. \& Bomsztyk, K. Protocol for the fast chromatin immunoprecipitation (ChIP) method. Nat. Protoc. 1, 179-185 (2006).

53. Dobin, A. et al. STAR: ultrafast universal RNA-seq aligner. Bioinformatics 29, 15-21 (2013).

54. Kipling, D. \& Kearsey, S. E. Reversion of autonomously replicating sequence mutations in Saccharomyces cerevisiae: creation of a eucaryotic replication origin within procaryotic vector DNA. Mol. Cell Biol. 10, 265-272 (1990).

\section{Acknowledgements}

We thank R. Allshire and K. Ishii for providing strains and reagents, J.W. Lee and Y.J. Choi for help with strain generation and bioinformatic analyses, and H.Yang for help with generating rabbit anti-CENP-A $\mathrm{A}^{\mathrm{Cnp1}}$ antiserum. This work was supported by grants from the Stem Cell Research Program (2012M 3A9B 4027953), Mid-career Researcher Program (2016R1A2B2006354), and Basic Science Research Program (2012R1A1A2008564) through the National Research Foundation of Korea (NRF), as well as the KAIST Future Systems Healthcare Project funded by the Ministry of Science, ICT, and Future Planning.

\section{Author contributions}

E.S.C. and Y.C. performed experiments and analyzed the data. K.K. helped initiate the ChIP-Seq analyses and contributed to data analyses. E.S.C., Y.C., and D.L. wrote and modified the manuscript.

\section{Additional information}

Supplementary Information accompanies this paper at doi:10.1038/s41467-017-00704-3.

Competing interests: The authors declare no competing financial interests.

Reprints and permission information is available online at http://npg.nature.com/ reprintsandpermissions/

Publisher's note: Springer Nature remains neutral with regard to jurisdictional claims in published maps and institutional affiliations.

(c) Open Access This article is licensed under a Creative Commons Attribution 4.0 International License, which permits use, sharing, adaptation, distribution and reproduction in any medium or format, as long as you give appropriate credit to the original author(s) and the source, provide a link to the Creative Commons license, and indicate if changes were made. The images or other third party material in this article are included in the article's Creative Commons license, unless indicated otherwise in a credit line to the material. If material is not included in the article's Creative Commons license and your intended use is not permitted by statutory regulation or exceeds the permitted use, you will need to obtain permission directly from the copyright holder. To view a copy of this license, visit http://creativecommons.org/ licenses/by/4.0/.

(C) The Author(s) 2017 